\title{
An Open-Label, Multicenter Observational Study for Patients with Alzheimer's Disease Treated with Memantine in the Clinical Practice
}

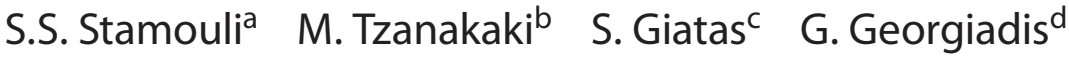 \\ E. Papalexi ${ }^{\mathrm{e}}$ I.A. Parashos ${ }^{\mathrm{e}}$
}

${ }^{\mathrm{a}}$ 1st Psychiatric Department, University of Athens, Eginition Hospital, Athens, ${ }^{\mathrm{b} C e n t e r}$ of Mental Health, Chania; 'Department of Neurology, 251 Military Hospital of Athens, Athens, ${ }^{d}$ Department of Neurology, Ippokratio General Hospital of Thessaloniki, Thessaloniki,

eLundbeck Hellas S.A., Athens, Greece

\section{Key Words}

Alzheimer's disease $\cdot$ Instrumental Activities of Daily Living $\cdot$ Memantine $\cdot$ Neurodegenerative disorders $\cdot$ Observational studies

\begin{abstract}
Background/Aims: In this post-marketing observational study, the safety and effectiveness of memantine were evaluated in patients with Alzheimer's disease (AD). Methods: In a 6-month, observational, open-label study at 202 specialist sites in Greece, the effectiveness of memantine was evaluated using the Mini-Mental State Examination (MMSE) and the Instrumental Activities of Daily Living (IADL) scale at baseline, and after 3 and 6 months. Discontinuation rates and adverse drug reactions (ADRs) were also recorded to evaluate the safety profile of memantine. Results: 2,570 patients participated in the study. Three and 6 months after baseline, MMSE and IADL scores were significantly improved compared to baseline. At the end of the study, $67 \%$ of the patients had improved their MMSE score; $7.1 \%$ of the patients reported $\geq 1$ ADRs, and treatment was discontinued due to ADR in $0.7 \%$. Conclusion: Memantine was well tolerated and had a positive effect on the patient's cognitive and functional ability in real-life clinical practice, in agreement with randomized, controlled trials.




\section{Introduction}

Alzheimer's disease (AD) is a progressive, extremely debilitating, neurodegenerative disorder, currently affecting almost 25 million people worldwide [1]. In 2002, the prevalence of dementia in Europe was estimated at 7 million individuals, and in most cases dementia was of the Alzheimer type [2,3]. It is estimated that by 2050, the number of individuals living with dementia will increase by $161 \%$ in developed countries [4]. Due to its symptomatology, $\mathrm{AD}$ is strongly associated with a significant burden on patients, caregivers and the society in general [5].

It has been well established that both the glutamatergic and the acetylcholinergic system work in synergy, contributing substantially to learning and memory, acquisition and retrieval $[6,7]$. In particular, glutamate, the main excitatory neurotransmitter in the central nervous system, is known to play a pivotal role in brain function, and thus memantine, a specific, moderate-affinity, uncompetitive N-methyl-D-aspartate (NMDA) receptor antagonist with strong voltage dependency and rapid blocking/unblocking kinetics [8, 9], has been approved and prescribed for the treatment of moderate to severe AD.

Treatment with memantine was associated with reduced clinical worsening in $A D$, based on an attempt to define marked clinical worsening as a decline of $\geq 4$ points on the Alzheimer's Disease Assessment Scale-Cognitive Subscale or $\geq 5$ points on the Severe Impairment Battery and any decline in the Clinician's Interview-Based Impression of Change Plus Caregiver Input and the Alzheimer's Disease Cooperative Study-Activities of Daily Living Inventory [10]. Recently published reviews/meta-analyses of randomized controlled trials (RCTs) with memantine treatment have shown that memantine reduces global, cognitive, and functional deterioration as well as the emergence of $\mathrm{AD}$-associated behavioral changes compared with placebo [11-16], and it is safe and well tolerated with an overall low rate of adverse effects $[17,18]$.

Although RCTs are undoubtedly the gold standard for drug evaluation, they do have certain limitations due to their strict inclusion/exclusion criteria, which may contribute to the underrepresentation of vulnerable patient groups $[19,20]$. Thus, observational studies could in some cases be a valuable source of supplementary data reflecting the use of a medicinal product in everyday clinical practice [19-21]. In the light of the above, the objective of the current observational study was to evaluate the effectiveness and tolerability of memantine when used in routine clinical practice in a large outpatient population suffering from $\mathrm{AD}$ in Greece.

\section{Methods}

In this open-label, multicenter, post-marketing observational study, $\mathrm{AD}$ patients were treated with memantine for 6 months. The study was conducted at 202 specialist sites. The patients were evaluated by psychiatrists and neurologists working for both the National Health Service, including 6 hospital outpatient units, and primarily private outpatient clinics in Greece.

\section{Patients}

No specific requirements were used for patient selection apart from the physician's positive diagnosis of $\mathrm{AD}$ based on clinical criteria. Also, contraindications and safety measures listed in the summary of product characteristics of memantine had to be noted. 


\section{Outcome Measurements}

The patients visited the clinic at the beginning of the study (baseline), and after 3 and 6 months of memantine treatment. At baseline, demographic data and information on previous anti-AD treatment were collected. At all 3 visits, cognitive and functional performance was assessed using the Mini-Mental State Examination (MMSE) [22] and the Instrumental Activities of Daily Living (IADL) [23] scales, respectively. The IADL scale consists of 8 items: ability to use the telephone, shopping, food preparation, housekeeping, laundry, mode of transportation, responsibility for own medications, and the ability to handle finances. The scale is ranging from 8 to 31, where ' 31 ' stands for total dependence and ' 8 ' for full autonomy. Each subitem is scored independently, ranging from 1 to 3, 4 or 5, depending on the subitem. Whereas in most cases women are scored on all 8 subitems of function, the areas of food preparation, housekeeping, and laundry are excluded for most men (scoring 0 which stands for 'not applicable'). In order not to exclude male patients, as has been done in other studies to avoid gender bias [24], the IADL point score was converted into a percentage score with higher percentage scores indicating greater functional impairment. To define the specific instrumental activities in which functional improvement was detected over time, the percentage of patients showing total independency (scoring ' 1 ' in the currently tested IADL subitem) and total dependency (scoring the highest mark in the currently tested IADL subitem) was recorded for each IADL subitem. Compliance was assessed by the investigator at each visit. Concomitant medication was monitored continuously during the study. Safety and tolerability was assessed throughout the study by collection of spontaneously reported adverse drug reactions (ADRs). In the case of premature study discontinuation, the reason for withdrawal was ascertained at the next scheduled visit (3 or 6 months).

\section{Statistical Analysis}

Descriptive statistics were used for baseline demographics and clinical characteristics. Multiple linear regression (MLR) and the appropriate univariate test were used to evaluate the relationship of the baseline MMSE and IADL scores with age or education (Pearson's correlation coefficient), sex or previous treatment ( $t$ test) and living conditions (ANOVA and Scheffé's test).

Analyses of MMSE and total IADL scores were performed for the intent-to-treat (ITT) population that included patients who had at least one evaluation after baseline and the perprotocol set (PPS) that included patients who had evaluations at all visits. Repeated-measures analysis of variance (ANOVA; Hotelling's test) and paired $t$ test were used to test differences in MMSE and IADL scores between visits. Missing values were entered using MLR with the 6-month score as dependent variable and the baseline/3-month score, age, and sex as independent variables. Wilcoxon signed-rank test was used to evaluate differences in the patient's ability to perform each single IADL item between the 1st and 3rd visits.

Improvement in the MMSE scale was defined as any change $>0$ scores on the 2 nd and 3rd visits compared to baseline, stabilization as no change and worsening as any change $<0$ (values of the scale of visit 2 or 3, respectively, minus values of the scale obtained at baseline). Taking the progressive neurodegenerative profile of the disease into consideration, the percentage of clinical significant worsening based on MMSE scores was also calculated (MMSE difference in scores $\geq 3$ points).

Improvement in IADL scores was defined as any difference $<0 \%$ between the 2 nd and 3 rd visits compared to baseline, stabilization as no change and worsening as any change $>0 \%$.

Descriptive statistics were used for safety and tolerability. MLR and the $\chi^{2}$ test were applied to investigate the relationship of treatment discontinuation and ADRs with age, sex, education level, living conditions, previous acetylcholinesterase inhibitor (AChEI) treatment, and current AD therapy. 


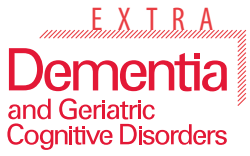

\begin{tabular}{l|l}
\hline Dement Geriatr Cogn Disord Extra 2011;1:10-19 \\
\hline \begin{tabular}{l|l} 
DOI: 10.1159/000322882 & $\begin{array}{l}\text { @ 2011 S. Karger AG, Basel } \\
\text { www.karger.com/dee }\end{array}$ \\
Published online: January 5, 2011 &
\end{tabular} \\
\hline
\end{tabular}

Table 1. Patient demographics and clinical characteristics

\begin{tabular}{|c|c|c|}
\hline & $\mathrm{n}$ & $\%$ \\
\hline \multicolumn{3}{|l|}{ Sex } \\
\hline Women & 1,402 & 54.6 \\
\hline Men & 1,168 & 45.4 \\
\hline \multicolumn{3}{|l|}{ Age } \\
\hline$\leq 65$ years & 192 & 7.5 \\
\hline $66-75$ years & 1,163 & 45.3 \\
\hline$>75$ years & 1,215 & 47.3 \\
\hline \multicolumn{3}{|l|}{ Educational level } \\
\hline 6 years & 1,409 & 54.8 \\
\hline 9 years & 638 & 24.8 \\
\hline 12 years & 248 & 9.6 \\
\hline Diploma & 123 & 4.8 \\
\hline University degree & 152 & 5.9 \\
\hline \multicolumn{3}{|l|}{ Living conditions } \\
\hline Alone & 263 & 10.2 \\
\hline With partner & 1,337 & 52.0 \\
\hline With children, relatives, or others & 893 & 34.7 \\
\hline In geriatric clinic & 77 & 3.0 \\
\hline \multicolumn{3}{|l|}{ Baseline MMSE (score) } \\
\hline Mild dementia (>20) & 1,095 & 42.6 \\
\hline Moderate dementia (10-19) & 1,314 & 51.1 \\
\hline Severe dementia $(<10)$ & 161 & 6.3 \\
\hline \multicolumn{3}{|l|}{ Previous treatment with AChEIs } \\
\hline Yes & 880 & 34.2 \\
\hline No & 1,690 & 65.8 \\
\hline \multicolumn{3}{|l|}{ Current treatment } \\
\hline Memantine & 2,080 & 80.9 \\
\hline $\begin{array}{l}\text { Memantine + AChEIs for the duration of } \\
\text { the study }\end{array}$ & 490 & 19.1 \\
\hline
\end{tabular}

\section{Results}

\section{Baseline Demographics and Clinical Characteristics}

In total, 2,570 patients participated in this study (table 1). At baseline, mean age $\pm \mathrm{SD}$ was 74.8 \pm 6.8 years and mean baseline MMSE score was 18.0 \pm 5.2 (MMSE range: 2-26). Mean baseline IADL score was $69.1 \pm 20.0 \%$. Of the AD patients, 1,690 (65.8\%) had never received therapy for dementia prior to their inclusion into the study, while the remaining $34.2 \%$ (880 patients) were already on AChEIs at baseline. During the study, 2,080 patients (80.9\%) were given memantine as monotherapy, while 490 patients (19.1\%) had combination therapy with AChEIs.

Regression and univariate analyses showed that the baseline MMSE score was negatively associated with age (MLR, Pearson correlation coefficient, $r=-0.245, p<0.001$ ), with older patients having lower baseline MMSE scores, being positively associated with educational level (MLR, Pearson correlation coefficient, $\mathrm{r}=0.147, \mathrm{p}<0.001$ ), and less-educated patients having lower baseline MMSE scores. Patients living alone or with their spouse had the highest baseline MMSE score, while patients living in a home care setting had the lowest baseline MMSE compared to all other groups (MLR, ANOVA and Scheffé test, $p<0.001$, for both comparisons). The baseline IADL score was negatively correlated with the baseline MMSE score (MLR, Pearson correlation coefficient, $r=-0.656, p<0.001$ ) and positively cor- 

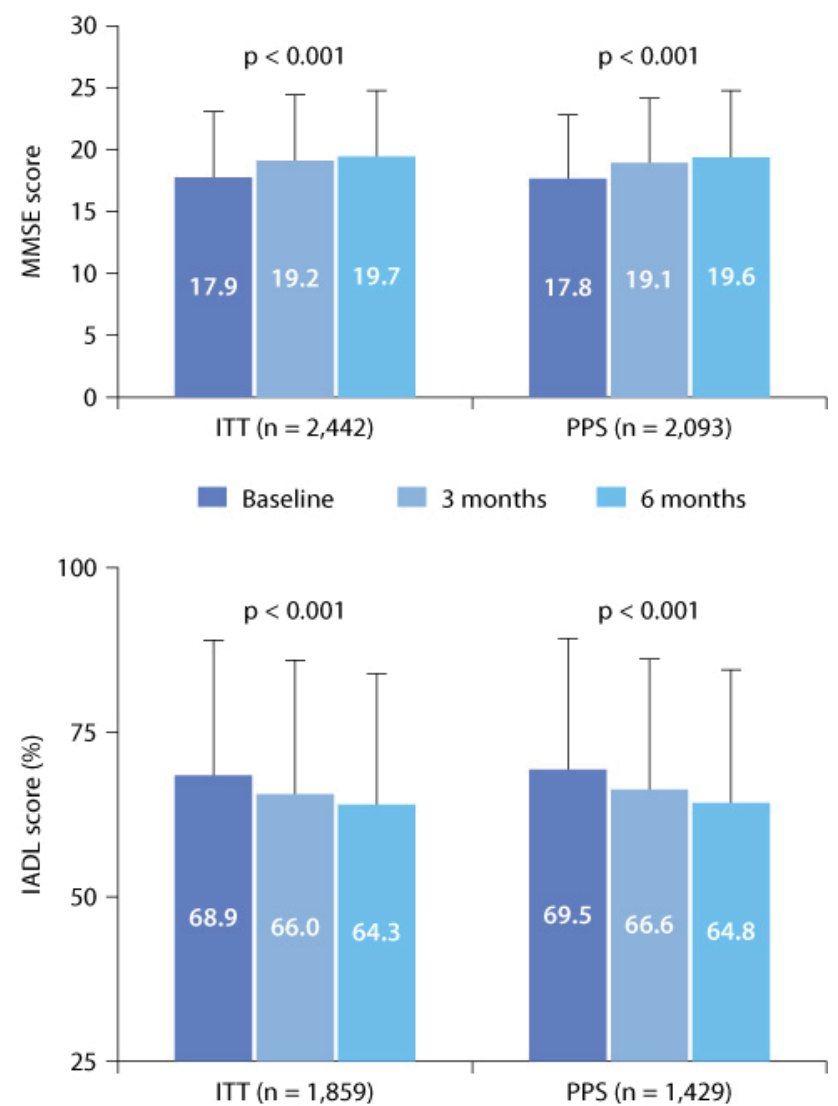

Fig. 1. Mean MMSE and IADL scores with standard deviations for the ITT and PPS populations. Overall $\mathrm{p}$ value (Hotelling's test): $\mathrm{p}<0.001$; and baseline vs. 3 months, baseline vs. 6 months, and 3 vs. 6 months (paired t test): $\mathrm{p}<0.001$.

related with age (MLR, Pearson correlation coefficient, $r=0.329, p<0.001$ ), with older patients having higher IADL scores and thus displaying less independency. Patients living alone had the lowest baseline IADL score compared to all other groups, and patients living with their spouse had lower baseline IADL scores than patients living with their children/ others or living in a home care setting (MLR, ANOVA and Scheffé test, $\mathrm{p}<0.001)$. Moreover, patients who had been treated with AChEIs previously had higher baseline IADL scores than untreated patients (MLR, $\mathrm{t}$ test, $\mathrm{p}<0.001)$.

\section{Effectiveness}

At 3 and 6 months, the MMSE score was significantly improved compared with the baseline score for the ITT population (repeated-measures ANOVA Hotelling's test, $n=2,442$ ). The same results were obtained in the PPS population (repeated-measures ANOVA Hotelling's test, $n=2,093$; fig. 1a). The paired-sample $t$ test detected statistically significant differences in each of the three visit pairs ( $\mathrm{p}<0.001$ for all three pairs, 1st visit vs. 2nd visit, 2 nd vs. 3rd visit, 1st vs. 3rd visit, for both ITT and PPS datasets). In the ITT population, $67.0 \%$ had improved their MMSE score, $18.6 \%$ had no change and $14.4 \%$ had worsening after 6 
Table 2. Withdrawal reasons

\begin{tabular}{lccc}
\hline & 1st to 2nd visit & 2nd to 3rd visit & Total \\
\hline Withdrawals, n (\%) & $140(5.4)$ & $347(13.5)$ & $487(18.9)$ \\
Adverse events, n (\%) & $15(0.6)$ & $4(0.1)$ & $19(0.7)$ \\
Treatment failure, n (\%) & $9(0.4)$ & $4(0.1)$ & $13(0.5)$ \\
Loss to follow-up, n (\%) & $87(3.4)$ & $316(12.3)$ & $403(15.7)$ \\
Patient's/caregiver's wish, n (\%) & $13(0.5)$ & $10(0.4)$ & $23(0.9)$ \\
Other reasons, n (\%) & $16(0.6)$ & $13(0.5)$ & $29(1.1)$ \\
\hline
\end{tabular}

months of treatment; 112 patients (4.6\%) experienced clinically significant worsening based on MMSE scores at the end of the study (decrease of $\geq 3$ points). Similar results were observed for the PPS population.

The total IADL percentage score was significantly improved after 3 and 6 months of memantine treatment both for the ITT (repeated-measures ANOVA Hotelling's test, $\mathrm{p}<0.001$, $\mathrm{n}=1,859$ ) and the PPS populations (repeated-measures ANOVA Hotelling's test, $\mathrm{p}<0.001$, $\mathrm{n}=1,429$; fig. 1b). The paired-sample $\mathrm{t}$ test detected statistically significant differences in each of the three visit pairs ( $\mathrm{p}<0.001$ for all three pairs, 1st vs. 2nd visit, 2nd vs. 3rd visit, 1st vs. 3rd visit, for both ITT and PPS datasets).

Statistical analysis of single IADL items using the Wilcoxon signed-rank test showed a statistically significant improvement for each IADL item, comparing the ability of patients to perform any specific instrumental activity at baseline and after 6 months of memantine treatment (Wilcoxon signed-rank test, $\mathrm{p}<0.001$, in all 8 IADL items).

\section{Safety and Tolerability}

During the current study, 487 (18.9\%) patients discontinued treatment (table 2). The most common reason leading to the majority of treatment discontinuations was loss to follow-up, while $<1 \%$ patients enrolled discontinued due to ADRs (table 2). The most common ADRs reported leading to treatment discontinuation were excitation (4 reports), somnolence (2 reports), constipation (2 reports), and nausea (2 reports).

Multiple logistic regression analyses (stepwise method) showed that patients living with children/relatives (21.6 vs. $14.8 \%$ living alone, $18 \%$ living with spouse and $19.5 \%$ living in a care home; $\mathrm{p}<0.05$ ), patients on monotherapy ( 21.1 vs. $9.8 \%$ for combination therapy; $\mathrm{p}<$ $0.001)$, and patients who had a higher baseline MMSE (21.7\% in mild cases versus $16.9 \%$ in moderate cases and $16.8 \%$ in severe cases; $\mathrm{p}<0.01$ ) were more likely to discontinue treatment.

Of the 2,570 patients, 21 were lost to follow-up before the 3-month visit, and consequently the safety population consisted of 2,549 patients. One or more ADRs were reported by 182 (7.1\%) patients (table 3). Dizziness and nausea were the most frequently reported ADRs, but still $<2 \%$ of the patients experienced either of these ADRs. ADRs of severe intensity occurred in 9 patients $(0.35 \%)$ : excitement (1 patient), bradycardia ( 1 patient, 2 reports), altered consciousness (2 patients), agitation (1 patient), nausea and agitation (1 patient), hypotension (1 patient), disinhibition (1 patient), and death (1 patient). According to the safety criteria, only 2 cases were recorded as serious ADRs (bradycardia and death). The patient who died was a 74-year-old man who developed oliguria and probably died because of acute renal failure. The patient had received memantine for almost 3 months. The patient's medical history comprised diabetes mellitus, a common cause of renal disease, which could have contributed to the event. The temporal sequence does not support causality, and the investigator who reported the case did not think that the event was related to memantine treatment. 
Table 3. ADRs occurring in $>1$ patient

\begin{tabular}{lll}
\hline ADRs & $\mathrm{n}$ & $\%$ \\
\hline Dizziness & 37 & 1.44 \\
Nausea & 33 & 1.28 \\
Headache & 23 & 0.89 \\
Excitement & 20 & 0.78 \\
Agitation & 11 & 0.43 \\
Somnolence & 8 & 0.31 \\
Gastrointestinal disorders & 6 & 0.23 \\
Constipation & 6 & 0.23 \\
Vomiting & 5 & 0.19 \\
Fatigue & 5 & 0.19 \\
Stomach ache & 4 & 0.16 \\
Imbalance & 4 & 0.16 \\
Diarrhea & 4 & 0.16 \\
Vertigo & 3 & 0.12 \\
Loss of appetite & 3 & 0.12 \\
Impaired consciousness & 3 & 0.12 \\
Sedation & 3 & 0.12 \\
Palpitations & 2 & 0.08 \\
Hypotonia & 2 & 0.08 \\
Insomnia & 2 & 0.08 \\
Tinnitus & 2 & 0.08 \\
Tremor & 2 & 0.08 \\
Dry mouth & 2 & 0.08 \\
\hline
\end{tabular}

\section{Discussion}

Due to the extremely strict inclusion and exclusion criteria of RCTs, experimental design trials are sometimes unable to provide answers to a number of clinically important questions $[19,20]$. The crucial difference between observational trials and RCTs is the absence of an experimental design. Thus, observational studies could be quite useful in studying already marketed pharmaceutical compounds in larger populations in comparison to RCTs, which may also include vulnerable patient groups [20]. Therefore, in the current open-label, postmarketing, observational study, memantine treatment was studied in a heterogeneous, large population of patients suffering from $\mathrm{AD}$ in order to assess its effectiveness and tolerability profile in naturalistic settings. The exclusion criteria were meant to be as minimal as possible, restricted only to the absence of $\mathrm{AD}$ diagnosis, in order to represent everyday medical practice as realistically as possible. Furthermore, the inclusion criteria were widely defined in order to obtain a typical sample size representing $\mathrm{AD}$ patients undergoing pharmacological treatment in real settings.

As far as safety issues are concerned, the reported frequency of total ADRs, as well as those of severe intensity, was found to be in alignment with both the documented frequency in the summary of product characteristics of memantine as well as with the published data derived from other observational studies on memantine conducted in Austria and Germany $[25,26]$. More specifically, in the German study, the ADR rate was < $5 \%$ [26]; in the Austrian 4 -month study, only $15.9 \%$ of patients reported an ADR [25]. The early discontinuation rate, which can be compared to the one from the Austrian study, was $18.9 \%$. The main reason for discontinuation was loss to follow-up visits, which could be attributed to different reasons, e.g. lack of effectiveness or non-reported ADRs. 
In accordance with already published clinical data as well as results from the aforementioned observational studies in other European countries [25, 26], memantine therapy resulted in statistically significant improvement or stabilization of the AD symptoms compared to baseline. The MMSE score was significantly improved compared to the baseline score at the two follow-up visits $(19.2 \pm 5.0$ vs. $17.9 \pm 5.1, \mathrm{p}<0.001$, and $19.7 \pm 5.1$ vs. 17.9 $\pm 5.1, \mathrm{p}<0.001$, respectively, for the ITT population, and $19.1 \pm 5.0$ vs. $17.8 \pm 5.1, \mathrm{p}<0.001$, and $19.6 \pm 5.1$ vs. $17.8 \pm 5.1, \mathrm{p}<0.001$, respectively, for the PPS population). Moreover, $67 \%$ of the patients showed an improvement and 19\% demonstrated no change in their MMSE score after 6 months of memantine treatment. This result is in agreement with the data for the MMSE score reported in the study by Calabrese et al. [26], in which $68.2 \%$ of the patients improved in the MMSE score by at least 1 point, and 9.6\% remained stable at the end of the study period.

Since $\mathrm{AD}$ is a progressive neurodegenerative disorder with no cure available to date and apart from symptom improvement the goal of $\mathrm{AD}$ treatment is the stabilization of the patient's condition, it was considered meaningful to calculate the percentage of patients experiencing significant clinical worsening at the end of the current 6-month study. In previously published reports based on the MMSE scale - a scale which has been broadly applied to evaluate the patients' cognitive symptoms and their severity evolution - it has been calculated that a patient receiving no treatment for dementia should lose approximately 3 points in the MMSE in 6 months [27].

Considering all the above, only $4.6 \%$ of the patients experienced clinically significant worsening based on MMSE scores at the end of the study.

Using the 8-item IADL scale allowing the assessment of changes in one's dependency over time, statistically significant improvement in IADL compared to baseline was reported for both the IADL score recorded as percentage as well as the single-item ADL analyses conducted at the end of the 6-month memantine treatment. More specifically, the IADL score was significantly improved compared to the baseline score (with lower scoring at the two follow-up visits, $66.0 \pm 19.7$ vs. $68.9 \pm 20.1, \mathrm{p}<0.001$, and $64.3 \pm 19.6$ vs. $68.9 \pm 20.1, \mathrm{p}<$ 0.001 , respectively, for the ITT population and $66.6 \pm 19.5$ vs. $69.5 \pm 20.0, \mathrm{p}<0.001$, and $64.8 \pm 19.7$ vs. $69.5 \pm 20.0, \mathrm{p}<0.001$, respectively, for the PPS population), showing less dependency in functional activities over time. In order to define the specific instrumental activities in which functional improvement was detected over time, the percentage of patients showing total independency and total dependency was recorded for each IADL subitem. It was evident that improvement was statistically significant in all eight instrumental activities evaluated by the IADL scale, with an increase in the percentage of patients able to perform each activity independently at each follow-up visit.

The present results concerning the functional ability of $\mathrm{AD}$ patients are in agreement with results from single-item ADL analyses [28] demonstrating the positive effect of memantine treatment on the maintenance or even improvement in the level of independency regarding daily activities such as shopping and food preparation. In other post-marketing studies [26], functional performance was also improved after a 6-month memantine treatment, although other scales were selected to evaluate activities of daily living (e.g. NOSGER - Nurses Observation Scale for Geriatric Patients). However, at a closer look, the improvement in the subscale of NOSGER in the observational study by Calabrese et al. [26] was in agreement with the current results, although their patients were at a more severe AD stage at baseline than the patients in the current study.

The results of this observational study should be seen and discussed after taking specific limitations into consideration. Besides the limitations of observational studies in general, mainly due to the absence of randomization and the lack of predefined endpoints, in the current study, the percentage of patients lost to follow-up visits was high. Most likely, this 
is due to the absence of close monitoring, which is applied in RCTs. The high percentage of patients lost to follow-up may have different reasons, including lack of effectiveness, disease progression, or occurrence of ADRs. Moreover, the Lawton IADL scale is not the most appropriate tool to evaluate response to dementia treatment and functional changes over time. However, the need for an easy-to-use and quickly administered scale led us to the decision to include it in the case report form of the observational study.

\section{Conclusion}

The results of this observational study in naturalistic settings support the outcomes demonstrated in RCTs of AD patients receiving memantine as treatment for dementia compared to placebo-treated patients, with $67 \%$ of the patients showing an improvement in their MMSE score at the end of the study and only $7.1 \%$ of patients reporting $\geq 1$ ADR throughout the study. The current results also seem to be in alignment with published results from other observational studies conducted in large AD patient cohorts treated with memantine.

\section{Acknowledgments}

The authors would like to thank all participating patients, caregivers and all investigators for their contribution to this study.

\section{Disclosure Statement}

E. Papalexi and I.A. Parashos are employees of Lundbeck Hellas S.A, Greece. This study was sponsored by Lundbeck Hellas.

\section{References}

-1 Ferri CP, Prince M, Brayne C, Brodaty H, Fratiglioni L, Ganguli M, Hall K, Hasegawa K, Hendrie H, Huang Y, Jorm A, Mathers C, Menezes PR, Rimmer E, Scazufca M, Alzheimer's Disease International: Global revalence of dementia: a Delphi consensus study. Lancet 2005;366:2112-2117.

-2 Waldemar G, Phung KT, Burns A, Georges J, Hansen FR, Iliffe S, Marking C, Rikkert MO, Selmes J, Stoppe G, Sartorius N: Access to diagnostic evaluation and treatment for dementia in Europe. Int J Geriatr Psychiatry 2007;22:47-54.

3 Lobo A, Launer LJ, Fratiglioni L, Andersen K, Di Carlo A, Breteler MM, Copeland JR, Dartigues JF, Jagger C, Martinez-Lage J, Soininen H, Hofman A: Prevalence of dementia and major subtypes in Europe: a collaborative study of population-based cohorts. Neurologic Diseases in the Elderly Research Group. Neurology 2000;54(11 suppl 5):S4-S9.

-4 Wimo A, Winblad B, Aguero-Torres H, von Strauss E: The magnitude of dementia occurrence in the world. Alzheimer Dis Assoc Disord 2003;17:63-67.

-5 Wimo A, Winblad B, Jönsson L: The worldwide societal costs of dementia: estimates for 2009. Alzheimers Dement 2010;6:98-103.

-6 Gold PE, Zornetzer SF: The mnemon and its juices: neuromodulation of memory processes. Behav Neural Biol 1983;38:151-189.

7 Francis PT: The interplay of neurotransmitters in Alzheimer's disease. CNS Spectr 2005;10(11 suppl $18): 6-9$.

-8 Parsons CG, Danysz W, Quack G: Memantine is a clinically well tolerated N-methyl-D-aspartate (NMDA) receptor antagonist - a review of preclinical data. Neuropharmacology 1999;38:735-767. 
-9 Parsons CG, Stoffler A, Danysz W: Memantine: a NMDA receptor antagonist that improves memory by restoration of homeostasis in the glutamatergic system - too little activation is bad, too much is even worse. Neuropharmacology 2007;53:699-723.

10 Wilkinson D, Andersen HF: Analysis of the effect of memantine in reducing the worsening of clinical symptoms in patients with moderate to severe Alzheimer's disease. Dement Geriatr Cogn Disord 2007;24:138-145.

-11 Winblad B, Jones R W, Wirth Y, Stoffler A, Mobius H J: Memantine in moderate to severe Alzheimer's disease: a meta-analysis of randomised clinical trials. Dement Geriatr Cogn Disord 2007;24: 20-27.

12 McShane R, Areosa Sastre A, Minakaran N: Memantine for dementia. Cochrane Database Syst Rev 2006;19:CD003154.

-13 Gauthier S, Loft H, Cummings J: Improvement in behavioural symptoms in patients with moderate to severe Alzheimer's disease by memantine: a pooled data analysis. Int J Geriatr Psychiatry 2008;23: 537-545.

-14 Wilcock GK, Ballard CG, Cooper JA, Loft H: Memantine for agitation/aggression and psychosis in moderately severe to severe Alzheimer's disease: a pooled analysis of 3 studies. J Clin Psychiatry 2008; 69:341-348.

-15 Mecocci P, Bladström A, Stender K: Effects of memantine on cognition in patients with moderate to severe Alzheimer's disease: post-hoc analyses of ADAS-cog and SIB total and single-item scores from six randomized, double-blind, placebo-controlled studies. Int J Geriatr Psychiatry 2009;24:532-538.

- 16 Emre M, Mecocci P, Stender K: Pooled analyses on cognitive effects of memantine in patients with moderate to severe Alzheimer's disease. J Alzheimers Dis 2008;14:193-199.

-17 Farlow MR, Graham SM, Alva G: Memantine for the treatment of Alzheimer's disease: tolerability and safety data from clinical trials. Drug Saf 2008;31:577-585.

18 Jones RW: A review comparing the safety and tolerability of memantine with the acetylcholinesterase inhibitors. Int J Geriatr Psychiatry 2010;25:547-553.

-19 Hannan EL: Randomized clinical trials and observational studies: guidelines for assessing respective strengths and limitations. JACC Cardiovasc Interv 2008;1:211-217.

-20 Lu CY: Observational studies: a review of study designs, challenges and strategies to reduce confounding. Int J Clin Pract 2009;63:691-697.

-21 Foody JM, Mendys PM, Liu LZ, Simpson RJ Jr: The utility of observational studies in clinical decision making: lessons learned from statin trials. Postgrad Med 2010;122:222-229.

-22 Folstein MF, Folstein SE, McHugh PR: Mini-mental state. A practical method for grading the cognitive state of patients for the clinician. J Psychiatr Res 1975;12:189-198.

-23 Lawton MP, Brody EM: Assessment of older people: self-maintaining and instrumental activities of daily living. Gerontologist 1969;9:179-186.

-24 Lechowski L, de Stampa M, Denis B, Tortrat D, Chassagne P, Robert P, Teillet L, Vellas B: Patterns of loss of abilities in instrumental activities of daily living in Alzheimer's disease: the REAL cohort study. Dement Geriatr Cogn Disord 2008;25:46-53.

25 Rainer M, Wuschitz A, Jagsch C: Memantine in everyday medical practice: beneficial effects in moderate to severe Alzheimer's disease. 9th Congress of the European Federation of Neurological Societies, Athens, September 17-20, 2005.

-26 Calabrese P, Essner U, Förstl H: Memantine (Ebixa) in clinical practice - results of an observational study. Dement Geriatr Cogn Disord 2007;24:111-117.

-27 Han L, Cole M, Bellavance F, McCusker J, Primeau F: Tracking cognitive decline in Alzheimer's disease using the mini-mental state examination: a meta-analysis. Int Psychogeriatr 2000;12:231-247.

-28 Feldman HH, Schmitt FA, Olin JT, Memantine MEM-MD-02 Study Group: Activities of daily living in moderate-to-severe Alzheimer disease: an analysis of the treatment effects of memantine in patients receiving stable donepezil treatment. Alzheimer Dis Assoc Disord 2006;20:263-268. 